\title{
Efectos marginales de los atributos de la tenencia de vivienda en el índice de pobreza multidimensional en Colombia durante el periodo 2013-2016
}

\author{
Marginal effects of housing tenure attributes in the multidimensional \\ poverty index in Colombia during the period 2013-2016
}

Diana Torres Martínez

dianatorresm@usantotomas.edu.co

\section{Resumen}

En la Declaración Universal de los derechos humanos se tienen aspectos diversos, todos en procura del bienestar de la humanidad. En coherencia con ello, los estados incluyen estos requerimientos globales en sus cartas de estado o constituciones. En esta dirección de la teoría del desarrollo humano, enmarcada en la Economía del bienestar, se brindan herramientas que amplían la visión sobre las distintas dimensiones del desarrollo humano, para que estas sean contempladas en las constituciones y puedan ser plenamente cumplidas en pro de la realización de los ciudadanos.

En esta investigación intervienen elementos propios del desarrollo humano, las necesidades básicas insatisfechas y el índice de pobreza multidimensional. Se plantea un recorrido teórico por cada uno de estos elementos y se vinculan entre sí por medio de un modelo econométrico donde la variable respuesta es el índice de pobreza. Dada la forma como en Colombia se mide este Índice, esta cobra real importancia a la hora de saber el estado de los habitantes del país y cuales podría ser los distintos caminos en términos de políticas estatales que puedan ir reduciendo esta condición de vulnerabilidad.

Palabras claves: Bienestar, Desarrollo Humano, Necesidades Básicas Insatisfechas, Índice de Pobreza Multidimensional.

\section{Abstract}

\footnotetext{
a Docente y Coordinadora de área de la Facultad de Estadística de la Universidad Santo Tomás. Estudió Licenciatura en Matemáticas en la Universidad Distrital y se graduó en el año 2005, posteriormente estudió Estadística en la Universidad Santo Tomás, recibiendo su título como profesional en Estadística en el año 2012. Es Magister de Ciencias Económicas en la Universidad Santo Tomás. Se desempeña como docente investigadora en la Universidad Santo Tomás en la facultad de Estadística y acompaña espacios académicos en lo referente a la probabilidad e inferencia estadística. dtorresm1013@gmail.com o dianatorresm@usantotomas.edu.co
} 
In the Universal Declaration of Human Rights there are diverse aspects all in pursuit of the welfare of humanity. In coherence with it, states include in their state letters or constitutions these global requirements. In this sense, the theory of human development, framed in the Welfare Economy, provides tools that broaden the vision of the different dimensions of human development, so that these are contemplated in the constitutions and can be fully complied with in favor of the realization of citizens.

This research involves elements of human development, unsatisfied basic needs and the multidimensional poverty index. A theoretical path is proposed for each of these elements and they are linked by an econometric model where the response variable is the poverty index. Given that the way how this Index is measured in Colombia, it takes on real importance at the time to know the state of the inhabitants of the country and what could be the different ways in terms of state policies that can reduce this condition of vulnerability.

Keywords: Wellbeing, Human Development, Unsatisfied Basic Needs, Multidimensional Poverty Index.

\section{Introducción}

El poseer una vivienda ha estado relacionado con una "buena" calidad de vida. Sin embargo, «en América Latina, la participación de las partidas destinadas a vivienda dentro del gasto social global, han sido tradicionalmente inferiores al resto de los sectores sociales» (Salas, 2001. p. 67). Esto impide alcanzar una cobertura total y adecuada de la vivienda para la población de cada país, manteniendo o aumentando así el déficit habitacional cuantitativo y cualitativo. En el Pacto Internacional de los Derechos Económicos Sociales y Culturales (PIDESC) (1996), en su artículo número 1 da relevancia a lo siguiente:

el derecho a una vivienda adecuada entendida como un lugar donde poderse aislar si se desea un espacio adecuado, seguridad adecuada, iluminación y ventilación adecuada, una infraestructura básica adecuada y una situación adecuada en relación con el trabajo y los servicios básicos, todo ello a un costo razonable.

No obstante, como lo plantea Escallón (2011) «lo importante no deberían ser sólo las cifras, sino garantizar que se construya ciudad, que se genere una oferta diversa y de calidad, para permitir el acceso de los más pobres a una solución de vivienda» (p. 58).

Ahora bien, es de interés de esta investigación abordar elementos como la pobreza y la tenencia de vivienda desde su déficit cualitativo, dado que el hecho de poseer un lugar para habitar no garantiza las "buenas" condiciones de éste; tampoco establece al propietario en condición de no de pobreza, ya que, en algunos casos, al pagar o adecuar la vivienda para constituirla como un espacio adecuado para habitarla, se pueden descuidar la consecución de elementos básicos de su canasta diaria. Es decir, «shelter 
induced poverty describes the situation where a household, after paying for housing (rent or mortgage plus utilities, property taxes and house maintenance), cannot afford the minimum poverty basket of non-housing goods» (Ruprah, 2010, p. 15). Esta situación es mucho más palpable en poblaciones con condiciones precarias, generando así un aumento en estos grupos de vulnerabilidad.

Por ello, la pregunta problémica, que es el hilo conductor de esta investigación, es: ¿cuáles han sido los efectos de la tenencia de vivienda y algunas características de los hogares en el Índice de Pobreza Multidimensional para Colombia en el periodo 20132016? En esta dirección, y a partir de esta inquietud, surge como sentencia o hipótesis de investigación la idea de que el Índice de Pobreza Multidimensional es causado por efectos de la tenencia de vivienda y algunas características de los hogares en el periodo 2013-2016.

El objetivo principal de esta investigación se plantea como: analizar e interpretar los efectos de la tenencia de vivienda y algunas características de los hogares en el Índice de Pobreza Multidimensional en Colombia, en periodo 2013- 2016, de modo que sea posible llevar a cabo recomendaciones de política pública que oriente una reducción del Índice de Pobreza Multidimensional. Para tal fin se han planteado los siguientes objetivos específicos:

Construir a partir de un marco de referencia, teórico, legal y conceptual que, de estructura a los ejes centrales de esta investigación, tal como lo son la pobreza y las capacidades de adquisición desde la perspectiva del desarrollo humano y la economía del bienestar vinculando esto con la medición de la pobreza por medio del índice de pobreza multidimensional establecido por la OPHI y medido en Colombia por el Departamento Nacional de Planeación. También mostrar un breve recorrido por las políticas públicas respecto a la tenencia de vivienda en los últimos años en Colombia.

En segundo lugar, se propone diseñar un modelo econométrico que permita evidenciar los efectos de la tenencia de vivienda y algunas características de los hogares en el Índice de Pobreza Multidimensional en Colombia, en el período 2013-2016. Para esto se hace uso de información secundaria tomada del Departamento Administrativo Nacional de Estadísticas DANE, específicamente en la medición de pobreza multidimensional.

En tercer lugar, se sugieren algunas recomendaciones adecuadas para la construcción de una política de intervención de vivienda en Colombia, teniendo en cuenta que, como lo dice Arcos (2018), «la vivienda es un bien privado, pero es objeto de políticas públicas, que, guardadas algunas diferencias, se ha orientado a privilegiar su consumo en ciertos sectores de la ciudad» (p. 55). Es probable que, siguiendo a García Norato (2007) «se requieran alternativas fundamentales en el desarrollo humano más que el económico, pues una vez que la persona logra el empoderamiento puede generar mayor desarrollo» (p. 270). Según los resultados hallados y los efectos de ésta en Índice de Pobreza Multidimensional. 


\section{Sección 1: Pobreza y calidad de vida}

Durante la revisión de investigaciones para este documento, se encontraron algunos elementos en común en investigadores que, desde sus experiencias, han escrito acerca de la pobreza y la calidad de vida de los individuos.

Por ejemplo, Maya y Bournazou (2012) analizan la relación la entre pobreza, marginalidad, desigualdad y la vivienda en el ámbito urbano en México y establecen una llamada "pobreza patrimonial". Ellos mencionan que la población que no tiene acceso a los mínimos parámetros aceptables de vivienda, vestido y transporte para cada uno de los miembros del hogar, sí cubren necesidades de alimentación, educación y salud.

Ante esta situación y su estrecha relación con las necesidades básicas insatisfechas, se puede evidenciar que, siguiendo el enfoque de las Necesidades Básicas Insatisfechas (NBI) (INE, 2002; Villarroel y Hernani-Limarino, 2013), se define la privación de una vivienda adecuada como la falta de al menos una de las siguientes cuatro características: (1) espacio adecuado (en relación al número de miembros de la familia y cuartos disponibles), (2) materiales adecuados en las paredes, (3) materiales adecuados en los pisos, y (4) materiales adecuados en el techo. Sin embargo, es importante mencionar que «aunque a primera vista se puede pensar que el acceso a una vivienda disminuye la pobreza de un hogar, no es claro de qué manera, por cuánto tiempo y a qué costos» (Camargo, 2011, p. 226).

Es decir, los gastos en mantenimiento y en algunas ocasiones en la disposición de las estrategias o programas estatales para el acceso a la vivienda generan responsabilidades adicionales para los individuos, tan marcadas y determinantes, que podría pensarse acerca de la dificultad en mantener una vivienda adecuada y digna. En consecuencia, «en América Latina, las tasas de pobreza e indigencia incrementan sustancialmente una vez se tiene en cuenta el gasto en vivienda» (Camargo, 2011. p. 234).

Pobreza y Desarrollo Humano

Cuando se habla de desarrollo normalmente se establece una relación proporcional y directa de éste con condiciones proporcionadas de la renta o ingreso de las personas en muchos casos de forma exclusiva. No obstante, esta condición de desarrollo de los individuos, según Sen (1999), no están relacionadas únicamente con el factor efectivo del ingreso sino con las capacidades, oportunidades y libertades de y para el mismo.

La libertad se presenta como las capacidades que posee una persona para llevar un tipo de vida que valoran con determinadas razones propias para valorar, el significado de vida se aleja del concepto de eternidad o de vivir por muchos años y se relaciona en lugar de un modo muy estrecho con «el vivir bien mientras se esté vivo (y no para vivir una vida de miseria y de privación de libertad)» (Sen, 1999, p. 30).

Este vivir bien estaría impregnado, no por las utilidades ni los bienes primarios solamente, sino más bien por las libertades fundamentales o capacidades para elegir la vida que queremos valorar, en donde, estos bienes primarios se convertirían en los medios 
para poner a prueba las capacidades de las personas de alcanzar sus fines deseados. Desde esta perspectiva «la calidad de vida depende de lo que el sujeto sea capaz de conseguir, de las maneras en que sea capaz de vivir, y no de su renta, disponibilidad de servicios sociales o satisfacción de necesidades básicas» (Cejudo, 2007, p. 11). O en palabras de Alkire (2002):

Consider how "dimensions" might relate to one approach to development, namely Amartya Sen's capability approach. In this approach, development is not defined as an increase in GNP per capita, or in consumption, health, and education measures alone, but as an expansion of capability. Capability refers to a person's or group's freedom to promote or achieve valuable functionings. (p. 184)

En esta dirección, se podría establecer una relación directa entre el desarrollo humano, dimensiones, capacidades y libertades. Respecto a estas últimas, Amartya Sen (2000) en su teoría de desarrollo humano establece los siguientes tipos de libertades: libertades políticas, servicios económicos, oportunidades sociales, garantías de transparencia y seguridad protectora.

Las libertades políticas, en forma de libertad de expresión y elecciones libres, contribuyen a fomentar la seguridad económica. Las oportunidades sociales, en forma de servicios educativos y sanitarios, facilitan la participación económica. Los servicios económicos, en formas de oportunidades para participar en el comercio y la producción, puede contribuir a generar riqueza personal, así como recursos públicos para financiar servicios sociales. (p. 28)

Ahora bien, tomando las capacidades como aquellas libertades de las personas que la hacer valorar el tipo de vida que lleva, la pobreza es fundamentada como la privación de estas capacidades básicas y no necesariamente se refiere a solo la falta de ingreso.

Iniciando la década de los noventa, el $\operatorname{PNUD}^{\mathrm{b}}$ (1992) definió el desarrollo como «el proceso de ampliar la gama de opciones de las personas, brindándoles mayores oportunidades de educación, atención médica, ingreso y empleo, y abarcando el espectro total de opciones humanas, desde un entorno físico en buenas condiciones hasta libertades económicas y políticas» (p. 18).

Hacia este tipo de desarrollo humano, desde el año 1990, anualmente se realiza un informe que coordina los esfuerzos nacionales e internacionales para alcanzar estos objetivos y llevar a cabo la medición de este desarrollo por medio de «tres elementos esenciales de la vida humana: longevidad, conocimientos y niveles decentes de vida» (PNUD, 1990, p. 36). Estos se miden de la siguiente manera:

- Longevidad: se mide por la esperanza de vida al nacer y se asume que entre más larga sea la vida, mayor es el bienestar para disfrutar y mayor la posibilidad de realizar los planes individuales.

${ }^{\mathrm{b}}$ El Programa de las Naciones Unidas para el Desarrollo (PNUD) fue fundado en 1965 con unos objetivos básicos, por un lado; la consecución del desarrollo humano y la lucha contra la pobreza. 
- Conocimientos: se mide por el porcentaje de adultos analfabetos, se asume que, a mayor educación, mayor criterio para trazar un proyecto de vida y mayores posibilidades de conseguirlo.

- Nivel de vida digna: se mide por la media entre los indicadores, acceso de servicios de salud y agua potable, porcentaje de niños desnutridos menores a 5 años e ingreso.

El desarrollo, para Sen, según Bedoya (2010):

debe ocuparse de mejorar la vida que llevan los individuos y las libertades que disfrutan. La expansión de las libertades que valoran enriquece su vida y la libera de restricciones, permitiendo que sean personas más plenas, que ejercen su propia voluntad, influyendo en el mundo en el que viven. (p. 286)

En este sentido, de acuerdo con Naciones Unidas, el desarrollo humano suele entenderse en primera instancia como la "construcción y disfrute" de una vida larga y saludable, con acceso al conocimiento y a los recursos necesarios para un nivel de vida decente.

Pobreza y Necesidades Básicas Insatisfechas

En este apartado se abordará la calidad de vida o el nivel de vida decente que dependerá de las posibilidades que tengan las personas de satisfacer de forma adecuada sus necesidades humanas fundamentales. En palabras de Álvarez y Martínez (2001): «las necesidades básicas insatisfechas se basan en juicios de valor sobre los niveles de bienestar mínimamente aceptables para llevar una vida digna y sobre todos los grados de privación que se consideran intolerables» (p. 30).

Estas necesidades estas relacionadas con indicadores simples seleccionados, con relación a los siguientes puntos según estos dos autores:

- Viviendas inadecuadas si la vivienda es móvil, refugio natural o puente, tiene pisos en tierra no tienen paredes o están hechas con tela o desechos.

- Viviendas con hacinamiento crítico si son viviendas donde viven hogares en los que hay más tres personas por cuarto.

- Viviendas con servicios inadecuados si la vivienda no tiene sanitario ni acueducto y su fuente de agua es un río, un carro tanque o agua lluvia.

- Viviendas con alta dependencia económica si son viviendas donde viven hogares con más de tres personas son la responsabilidad de un jefe de hogar con trabajo, cuyo nivel de educación es de tres años o menos.

- Viviendas con niños en edad escolar que no asisten a la escuela si son viviendas donde viven hogares en el que hay al menos un niño mayor de seis años y menor que doce que no asiste a un centro de educación formal.

Según el DANE, la metodología de NBI busca determinar, con ayuda de algunos indicadores simples, si las necesidades básicas de la población se encuentran cubiertas. Los grupos que no alcancen un umbral mínimo fijado, son clasificados como pobres. 
Ahora bien, para Max-Neef (1993) las necesidades humanas pueden distinguirse según varios criterios para este autor desde categorías existenciales (relacionadas con el ser, tener, hacer y estar) y categorías axiológicas, tales como:

la subsistencia (debidas a la alimentación y abrigo insuficientes); de protección (debida a los sistemas de salud insuficientes, a la violencia, entre otras); de afecto (debidas a la opresión, relaciones de explotación con el medio ambiente natural, entre otras); de entendimiento (debido a la deficiente calidad de la educación); de participación (debido a la marginación y discriminación); de identidad (debido a imposición de valores extraños, emigración forzada, entre otras). (p. 43)

Es posible conocer que en estas necesidades manifiestas se encuentran ciertos elementos relacionados con el tener un lugar de abrigo y que éste tenga una habitabilidad adecuada, así como el tener algunas de las necesidades básicas cubiertas, brinda mayor posibilidad a los individuos de tener mejor calidad de vida.

\section{Pobreza e Índice de Pobreza Multidimensional}

El Índice de Pobreza Multidimensional al que este estudio se refiere es el calculado por el DNP con el apoyo técnico de la $\mathrm{OPHI}^{\mathrm{c}}$, con la metodología de Alkire y Foster. Según estos autores: «How we measure poverty can importantly influence how we come to understand it, how we analyze it, and how we create policies to influence it. For this reason, measurement methodologies can be of tremendous practical relevance» (Alkire y Foster, 2011, p. 290).

En concordancia con Amartya Sen (2000), un concepto de pobreza debe incluir dos ejercicios bien definidos, estos son la identificación, un método para incluir a un grupo de personas en la categoría de pobres, y la agregación, un método para integrar las características del conjunto de pobres en una imagen global de la pobreza.

En el Índice de Pobreza Multidimensional, Alkire y Foster (2011) establecen en su planteamiento dos enfoques para la identificación: unión e intersección. En la identificación por unión, una persona privada de cualquier dimensión se considera pobre; ahora, bajo identificación de intersección, solo las personas privadas de todas las dimensiones se consideran pobres. Sin embargo, este último enfoque podría ser particularmente ineficaz para separar a los pobres de los no pobres, ya que los mismos autores mencionan un estudio previo suyo en el que utilizaron diez dimensiones para identificar a los pobres en India. En este el enfoque de unión identificó al 97\% de la

\footnotetext{
${ }^{c}$ OPHI corresponden a las siglas en inglés de La Iniciativa de Desarrollo Humano y Reducción de la Pobreza. Está compuesto por un equipo de investigadores de diversas nacionalidades, y tiene como asesores y colaboradores a importantes académicos, como ser Amartya Sen, Tony Atkinson, Sudhir Anand, Frances Stewart y James Foster.
} 
población como pobre, una sobrestimación de la pobreza, mientras que el enfoque de intersección identificó una décima parte de 1\%, una subestimación de la pobreza.

En los pasos seguidos para el cálculo del Índice, Alkire y Foster (2011) establecen, en primer lugar, la definición de la unidad de análisis, bien sea individuos u hogares, luego se hace la elección de las dimensiones involucradas y las variables o los indicadores para cada una de las dimensiones.

Respecto a los umbrales de privación, estos se establecen por cada una de las dimensiones y, si la persona no alcanza el límite de privación respectivo, se dice que la persona está privada de esa dimensión; si el nivel de la persona es al menos tan grande como el límite de privación, la persona no se ve privada.

Para establecer los pesos de cada dimensión, se crea un vector de valores de privación que indica la importancia relativa de las diferentes privaciones. Aquí es importante mencionar que los valores de privación afectan a la identificación, ya que determinan las combinaciones mínimas de privaciones que identificarán a una persona como pobre; también afectan la agregación al alterar las contribuciones relativas de las privaciones a la pobreza general. Posteriormente, se lleva a cabo un conteo de privación que refleja la amplitud de la privación de cada unidad de análisis. El recuento de privación de la iésima unidad ci es el número de privaciones experimentadas por i (en el caso de pesos iguales), o la suma de los valores de las privaciones experimentadas por i (en el caso general).

Ahora bien, para crear un umbral de pobreza se establece $\mathrm{k}$, que satisface $0<\mathrm{k} \leq \mathrm{d}$, usado para determinar si una unidad de análisis tiene privaciones suficientes para ser considerado pobre. Si la cuenta de privación de la i-ésima unidad ci cae por debajo de k, esta no se considera pobre; si el conteo de privación de la unidad es k o superior, la unidad se identifica como pobre.

Por último, se lleva a cabo una identificación de quién es pobre bajo la óptica multidimensional y se definen los métodos de agregación dentro y a través de las dimensiones.

Estos métodos de agregación se establecen bajo la línea de corte dual lo que implica una familia de índices que permite la medición de la incidencia, la incidencia ajustada, la brecha y la severidad.

\section{Sección 2: En Colombia el IPM}

Para la medición de la pobreza por medio del Índice de Pobreza Multimensional (IPM), en Colombia inicialmente se lleva a cabo una identificación de las personas pobres utilizando un método de "corte dual. Para ello, según en DNP (2011), se establece un punto de corte en cada dimensión para identificar las personas con privación en cada dimensión; luego, se define un punto de corte que determina el número de privaciones que debe enfrentar una persona para ser considerada pobre. El método de corte dual es especialmente útil cuando el número de dimensiones es grande e incluye como soluciones particulares el enfoque de unión y el de intersección. En suma, este método permite 
establecer un punto de corte intermedio entre al menos una privación y todas las privaciones. Para los autores, una persona es identificada como pobre cuando sufre privaciones en al menos k dimensiones. En relación con lo anterior, es importante mencionar que no existe un método determinístico para definir el parámetro k.

El IPM mide la pobreza a través de 5 dimensiones que involucran 15 indicadores, los cuales son obtenidos por medio de la Encuesta Nacional de Calidad de Vida. Los indicadores utilizados y expuestos por Ángulo (2011) y por el DNP son los siguientes:

Condiciones educativas

Logro educativo: una persona se considera privada si pertenece a un hogar donde la educación promedio de las personas mayores de 15 años es menor a 9 años de educación.

Analfabetismo: una persona se considera privada si pertenece a un hogar en el que hay al menos una persona de 15 años y más que no sabe leer y escribir.

Condiciones de la niñez y juventud

Asistencia escolar: una persona se considera privada si pertenece a un hogar que tiene al menos un niño entre 6 y 16 años que no asiste a una institución educativa.

Rezago escolar: una persona se considera privada si pertenece a un hogar que tiene al menos hay un niño entre 7 y 17 años con rezago escolar (número de años aprobados inferior a la norma nacional).

Acceso a servicios para el cuidado de la primera infancia: una persona se considera privada si pertenece a un hogar que tiene al menos un niño de 0 a 5 años sin acceso a todos los servicios de cuidado integral (salud, nutrición y cuidado).

Trabajo infantil: una persona se considera privada si pertenece a un hogar que tiene al menos un niño entre 12 y 17 años trabajando.

Trabajo

Desempleo de larga duración: una persona se considera privada si pertenece a un hogar que tiene al menos una persona de la Población Económicamente Activa (PEA) desempleada por más de 12 meses.

Empleo formal: una persona se considera privada si pertenece a un hogar que tiene al menos un ocupado que no tiene afiliación a pensiones o se encuentra en desempleo.

Salud

Aseguramiento en salud: una persona se considera privada si pertenece a un hogar que tiene al menos una persona mayor de 5 años que no se encuentre asegurada en salud.

Servicio de salud dada una necesidad: una persona se considera privada si pertenece a un hogar que tiene al menos una persona que en los últimos 30 días tuvieron una enfermedad, accidente, problema odontológico o algún otro problema de salud que no haya implicado hospitalización y que para tratar este problema de salud no acudieron a 
un médico general, especialista, odontólogo, terapista o institución de salud.

Servicios públicos y condiciones de la vivienda

Acceso a fuente de agua mejorada: una persona se considera privada si pertenece a un hogar que no cuenta con servicio de acueducto.

Eliminación de excretas: una persona se considera privada si pertenece a un hogar que no cuenta con servicio público de alcantarillado.

Pisos: una persona se considera privada si pertenece a un hogar cuya vivienda cuenta con pisos de tierra.

Paredes exteriores: una persona se considera privada si pertenece a un hogar cuya vivienda cuenta con paredes de madera burda, tabla, tablón, guadua, otro vegetal, zinc, tela, cartón, desechos y sin paredes.

Hacinamiento crítico: una persona se considera privada si pertenece a un hogar donde hay 3 o más personas por cuarto.

Según en DNP, la construcción de Índice de Pobreza Dimensional se hizo con base en la metodóloga de Alkire y Foster (2007), mencionada previamente y cuyas principales ventajas radican en:

a. No sólo es un indicador de incidencia sino de brecha y severidad.

b. Cumple con las propiedades axiomáticas deseables en una medida de pobreza establecidas por Amartya Sen $(1976,1979)$.

c. Permite hacer un seguimiento de política pública puesto que la definición de las variables determina el responsable de su cumplimiento.

d. Se constituye en un instrumento que permite la focalización de la política en grupos poblaciones con múltiples carencias, exaltando su utilidad en la medida en que permite suplir las carencias a las que se enfrentan los más necesitados.

La metodología usada consiste en construir una matriz de 1 o 0 , donde el 1 representa privación y 0 no privación. En la matriz las filas representan los hogares y las columnas las 15 variables evaluadas en este caso. Una vez construida la matriz de privaciones por hogar se procede a construir la variable IPM al realizar una suma ponderada de la matriz. Finalmente, un hogar se cataloga como "pobre", según IPM, si el índice ponderado es superior o igual a, un tercio de las variables consideradas.

Los valores de este indicador en los últimos años se muestran a continuación en la figura No 1. 


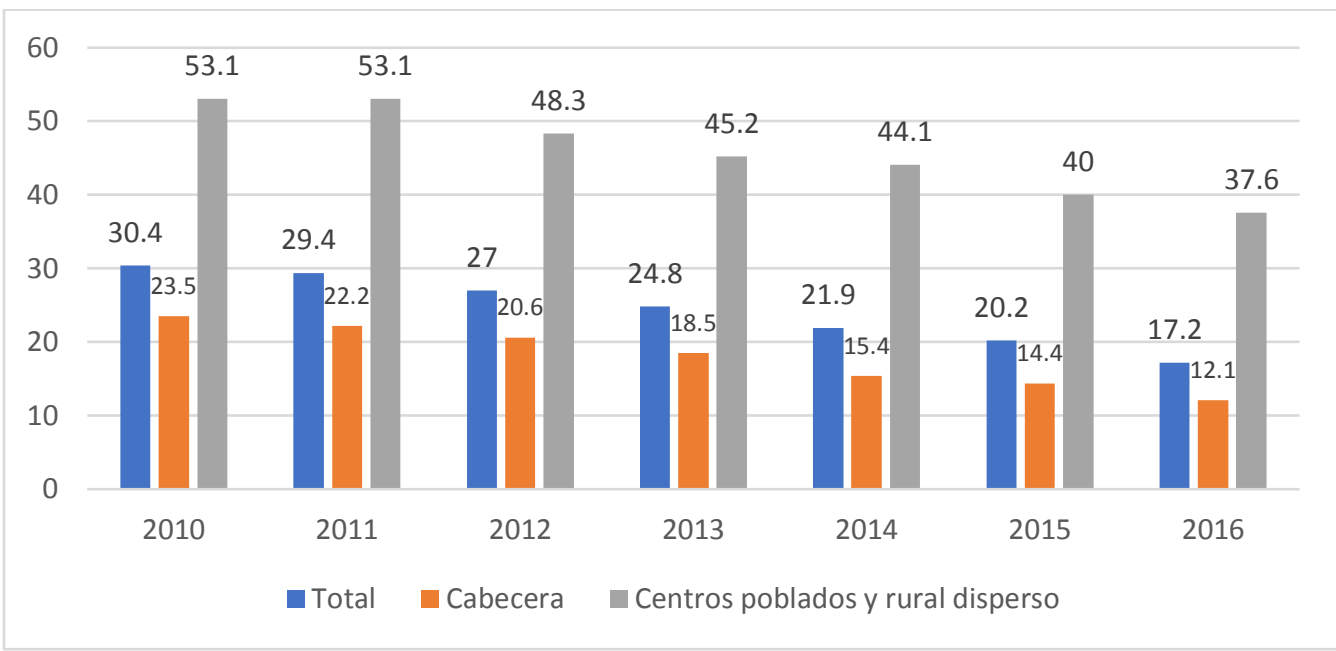

Figura No 1: Índice de Pobreza Multidimensional 2010-2016

Fuente: DNP

En la Figura No 1 se observa que se ha presentado una reducción del IPM del año 2010 hasta lo registrado en el año 2016, con una variación mayor en los años 2013 y 2014 de 2,9 puntos porcentuales.

\section{Sección 3: Modelo Econométrico}

A continuación, se describe la modelización del Indicé de Pobreza Multidimensional por medio de una metodología estadística y econométrica:

Variables incluidas en el modelo

Los cálculos para este trabajo se realizaron a partir del los microdatos registrados en el Índice de Pobreza Multidimensional (IPM) en el Departamento Nacional de Estadísticas DANE, el cual ha tomado estos datos a partir de su Encuesta de Calidad de Vida (ECV) para el periodo 2013-2016.

Las variables que se tomarán como endógenas en el modelo econométrico de este trabajo se describen en la tabla No 1.

Tabla No: 1 Variables incluidas en el modelo

\begin{tabular}{|c|c|c|}
\hline \multicolumn{2}{|r|}{ VARIABLE } & DESCRPCIÓN \\
\hline 1. & $\mathrm{P} 5010$ & No de cuartos en la vivienda. Discreta \\
\hline 2. & PERSONAS & No de personas en el hogar. Discreta \\
\hline 3. & LOGRO_ED & $\begin{array}{l}\text { Privación por Bajo Logro Educativo. Cualitativa. } \\
\text { (0: No privación, 1: Privación) }\end{array}$ \\
\hline 4. & INASISTE & $\begin{array}{l}\text { Privación por Inasistencia Escolar. Cualitativa. } \\
\text { (0: No privación, 1: Privación) }\end{array}$ \\
\hline 5 . & DESEMPLE & $\begin{array}{l}\text { Privación por Desempleo de Larga Duración. } \\
\text { Cualitativa. (0: No privación, 1: Privación) }\end{array}$ \\
\hline
\end{tabular}




\begin{tabular}{|ll|l|}
\hline 6. & ASEGURAM & $\begin{array}{l}\text { Privación por no aseguramiento en salud. } \\
\text { Cualitativa. (0: No privación, 1: Privación) }\end{array}$ \\
\hline 7. & REGION & Región. Cualitativa. \\
\hline 8. & ACUEDUCT & $\begin{array}{l}\text { Privación por no acceso a fuente de agua } \\
\text { mejorada. Cualitativa. (0: No privación, 1: } \\
\text { Privación) }\end{array}$ \\
\hline 9. & ALCANTAR & $\begin{array}{l}\text { Privación por inadecuada privación de excretas. } \\
\text { Discreta Numérica. (0: No privación, 1: } \\
\text { Privación) }\end{array}$ \\
\hline 10. & PISOS & $\begin{array}{l}\text { Privación por inadecuado material de pisos. } \\
\text { Cualitativa. (0: No privación, 1: Privación) }\end{array}$ \\
\hline 11. & PAREDES & $\begin{array}{l}\text { Privación por inadecuado material de paredes } \\
\text { externas. Cualitativa. (0: No privación, 1: } \\
\text { Privación) }\end{array}$ \\
\hline 12. & HACINAMI & $\begin{array}{l}\text { Privación por hacinamiento crítico. Cualitativa. } \\
\text { (0: No privación, 1: Privación) }\end{array}$ \\
\hline 13. & P6040 & Edad del jefe de hogar (años). Continua. \\
\hline 14. & P6160 & Sabeleer y escribir. Cualitativa. (1: Sí, 2: No) \\
\hline 15. & P8586 & Estudia. Discreta Cualitativa. (1: Sí, 2: No) \\
\hline 16. & P6920 & $\begin{array}{l}\text { Cotiza a un fondo de pensiones. Cualitativa. (1:Si, } \\
2: \text { No, 3: Ya es pensionado) }\end{array}$ \\
\hline 17. & P4005 & $\begin{array}{l}\text { Material predominante en las paredes exteriores. } \\
\text { Cualitativa }\end{array}$ \\
\hline
\end{tabular}

Fuente: Dane

Descriptivos de la variable IPM

En la tabla No 2, los estadísticos de la variable endógena Índice de Pobreza Multidimensional muestran que la tendencia de este índice se centra alrededor del 0,25, siendo en el año 2016 el registro más bajo de este tiempo. Para el año 2013, el mayor índice del $25 \%$ más bajo es 0,17 y el más bajo de la cuarta parte de índices más altos es 0,34. Para el año 2016, del $25 \%$ de los hogares con los menores índices, aquel que cuenta el máximo lo registra en 0,15 y del $75 \%$ de hogares con mayor índice el menor registro está en 0,3 .

Tabla No 2: Estadísticos IPM

\begin{tabular}{|c|c|c|c|c|}
\hline Estadísticos & 2013 & 2014 & 2015 & 2016 \\
\hline $\begin{array}{c}\text { Tamaño de muestra } \\
\text { No de hogares }\end{array}$ & 20167 & 18838 & 21.183 & 21.087 \\
\hline $\begin{array}{c}\text { Tamaño de } \\
\text { población } \\
\text { (Aplicando Factor } \\
\text { de expansión) } \\
\text { No de hogares }\end{array}$ & 12.190 .907 & 12.444 .475 & 12.504 .820 & 13.349 .505 \\
\hline
\end{tabular}

Comunicaciones en Estadística Junio 2019, Vol. 12, No. 1 


\begin{tabular}{|c|r|r|r|r|}
\hline Media & 0,2542 & 0,2498 & 0,2432 & 0,2355 \\
\hline Mediana & 0,25 & 0,24 & 0,24 & 0,2 \\
\hline Moda & 0,2 & 0,2 & 0,2 & 0,2 \\
\hline Desviación estándar & 0,1203 & 0,1173 & 0,1172 & 0,1144 \\
\hline Máximo & 0,82 & 0,78 & 0,82 & 0,72 \\
\hline Mínimo & 0,04 & 0,04 & 0,04 & 0,04 \\
\hline Percentil 25 & 0,17 & 0,15 & 0,15 & 0,15 \\
\hline Percentil 75 & 0,34 & 0,33 & 0,3 & 0,3 \\
\hline
\end{tabular}

Fuente: Elaboración propia con microdatos del Dane

Porcentaje de privación de variables relacionadas con el IPM

Ahora bien, dentro de este estudio se incluyen 9 de esas 15 variables tomadas por el DNP, de las cuales se observa que respecto la privación por bajo logro educativo se ha mantenido en estos 4 años una misma tendencia. En el año 2016 se presentó el 60,9\%; el mayor porcentaje frente a este tipo de privación.

Con relación a la privación por inasistencia escolar, este es radicalmente menor al de la privación anterior. El año en que se presentó con mayor porcentaje fue en el 2013 con 4,1\%. Tabla No 3 .

Tabla No 3: Condiciones educativas y condiciones de la niñez y juventud. (No de hogares)

\begin{tabular}{|c|r|r|r|r|r|}
\hline \multirow{2}{*}{ Año } & \multicolumn{2}{|c|}{ Bajo logro educativo } & \multicolumn{2}{c|}{ Inasistencia escolar } & \multirow{2}{*}{ TOTAL } \\
& No privación & Privación & \multicolumn{1}{c|}{$\begin{array}{c}\text { No } \\
\text { privación }\end{array}$} & Privación & \\
\hline \multirow{2}{*}{2013} & 5.262 .856 & 6.928 .051 & $\begin{array}{r}11.685 .518 \\
(95,9 \%)\end{array}$ & 505.389 & $(4,1 \%)$ \\
& $(43,2 \%)$ & $(56,8 \%)$ & $\begin{array}{r}12.190 .908 \\
(100 \%)\end{array}$ \\
\hline \multirow{2}{*}{2014} & 5.403 .557 & 7.040 .917 & 11.983 .395 & 461.080 & 12.444 .475 \\
& $(43,4 \%)$ & $(56,6 \%)$ & $(96,3 \%)$ & $(3,7 \%)$ & $(100 \%)$ \\
\hline 2015 & 5.289 .685 & 7.215 .134 & 12.084 .166 & 420.653 & 12.504 .820 \\
& $(42,3 \%)$ & $(57,7 \%)$ & $(96,6 \%)$ & $(3,4 \%)$ & $(100 \%)$ \\
\hline 2016 & 5.224 .862 & 8.124 .642 & 12.843 .436 & 506.069 & 13.349 .505 \\
& $(39,1 \%)$ & $(60,9 \%)$ & $(96,2 \%)$ & $(3,8 \%)$ & $(100 \%)$ \\
\hline
\end{tabular}

Fuente: Elaboración propia con microdatos del Dane

En las privaciones respecto al trabajo y la salud y en relación al desempleo de larga duración, la mayor cantidad de hogares que reportan esta privación corresponde al año 2015, con 12,3\%. Acerca del aseguramiento en salud fue el año 2013 en el que más se registró esta situación de privación con $18,8 \%$. Ver Tabla No 4. 
Tabla No 4: Trabajo y Salud

\begin{tabular}{|c|c|c|c|c|c|}
\hline \multirow[t]{2}{*}{ Año } & \multicolumn{2}{|c|}{$\begin{array}{c}\text { Desempleo de larga } \\
\text { duración }\end{array}$} & \multicolumn{2}{|c|}{ Aseguramiento en salud } & \multirow[t]{2}{*}{ TOTAL } \\
\hline & No privación & Privación & $\begin{array}{c}\text { No } \\
\text { privación }\end{array}$ & Privación & \\
\hline 2013 & $\begin{array}{r}10.891 .622 \\
(89,3 \%) \\
\end{array}$ & $\begin{array}{r}1.299 .285 \\
(10,7 \%) \\
\end{array}$ & $\begin{array}{r}9.900 .040 \\
(81,2 \%) \\
\end{array}$ & $\begin{array}{r}2.290 .867 \\
(18,8 \%) \\
\end{array}$ & $\begin{array}{r}12.190 .908 \\
(100 \%) \\
\end{array}$ \\
\hline 2014 & $\begin{array}{r}11.006 .105 \\
(88,4 \%)\end{array}$ & $\begin{array}{r}1.438 .369 \\
(11,6 \%)\end{array}$ & $\begin{array}{r}10.756 .101 \\
(86,4 \%)\end{array}$ & $\begin{array}{r}1.688 .374 \\
(13,6 \%)\end{array}$ & $\begin{array}{r}12.444 .475 \\
(100 \%)\end{array}$ \\
\hline 2015 & $\begin{array}{r}10.967 .906 \\
(87,7 \%) \\
\end{array}$ & $\begin{array}{r}1.536 .913 \\
(12,3 \%) \\
\end{array}$ & $\begin{array}{r}10.923 .474 \\
(87,4 \%) \\
\end{array}$ & $\begin{array}{r}1.581 .346 \\
(12,6 \%) \\
\end{array}$ & $\begin{array}{r}12.504 .820 \\
(100 \%) \\
\end{array}$ \\
\hline 2016 & $\begin{array}{r}11.727 .564 \\
(87,9 \%)\end{array}$ & $\begin{array}{r}1.621 .941 \\
(12,1 \%)\end{array}$ & $\begin{array}{r}11.935 .526 \\
(89,4 \%)\end{array}$ & $\begin{array}{r}1.413 .979 \\
(10,6 \%)\end{array}$ & $\begin{array}{r}13.349 .505 \\
(100 \%)\end{array}$ \\
\hline
\end{tabular}

Fuente: Elaboración propia con microdatos del Dane

Desde las privaciones relacionadas con los servicios públicos y las condiciones de la vivienda que se muestran en las siguientes tablas, se evidencia, respecto al acceso a fuente de agua mejorada, que la privación mayor se encuentra en el año 2016, con un $19,6 \%$. Ver Tabla No 5 .

Tabla No 5: Servicios públicos y condiciones de vivienda

\begin{tabular}{|c|c|c|c|c|c|}
\hline \multirow[t]{2}{*}{ Año } & \multicolumn{2}{|c|}{$\begin{array}{c}\text { Acceso a fuente de agua } \\
\text { mejorada }\end{array}$} & \multicolumn{2}{|c|}{$\begin{array}{c}\text { Alcantarillado } \\
\text { (eliminación de excretas) }\end{array}$} & \multirow[t]{2}{*}{ TOTAL } \\
\hline & No privación & Privación & $\begin{array}{l}\text { No } \\
\text { privación }\end{array}$ & Privación & \\
\hline 2013 & $\begin{array}{r}10.685 .298 \\
(87,6 \%)\end{array}$ & $\begin{array}{r}1.505 .609 \\
(12,4 \%)\end{array}$ & $\begin{array}{r}10.611 .635 \\
(87 \%)\end{array}$ & $\begin{array}{r}1.579 .272 \\
(13 \%)\end{array}$ & $\begin{array}{r}12.190 .908 \\
(100 \%)\end{array}$ \\
\hline 2014 & $\begin{array}{r}10.862 .941 \\
(87,3 \%)\end{array}$ & $\begin{array}{r}1.581 .534 \\
(12,7 \%)\end{array}$ & $\begin{array}{r}10.996 .507 \\
(88,4 \%)\end{array}$ & $\begin{array}{r}1.447 .968 \\
(11,6 \%)\end{array}$ & $\begin{array}{r}12.444 .475 \\
(100 \%)\end{array}$ \\
\hline 2015 & $\begin{array}{r}10.844 .646 \\
(86,7 \%) \\
\end{array}$ & $\begin{array}{r}1.660 .173 \\
(13,3 \%) \\
\end{array}$ & $\begin{array}{r}10.974 .049 \\
(87,8 \%) \\
\end{array}$ & $\begin{array}{r}1.530 .770 \\
(12,2 \%) \\
\end{array}$ & $\begin{array}{r}12.504 .820 \\
(100 \%)\end{array}$ \\
\hline 2016 & $\begin{array}{r}10.736 .346 \\
(80,4 \%) \\
\end{array}$ & $\begin{array}{r}2.613 .159 \\
(19,6 \%) \\
\end{array}$ & $\begin{array}{r}11.023 .162 \\
(82,6 \%) \\
\end{array}$ & $\begin{array}{r}2.326 .343 \\
(17,4 \%) \\
\end{array}$ & $\begin{array}{r}13.349 .505 \\
(100 \%) \\
\end{array}$ \\
\hline
\end{tabular}

Fuente: Elaboración propia con microdatos del Dane

En cuanto al servicio de alcantarillado, es decir, la eliminación adecuada de excretas, también es el año 2016 donde se encuentra una mayor cantidad de hogares respecto a esta privación, 17,4\%. Ver Tabla No 6. 
Tabla No 6: Servicios públicos y condiciones de vivienda

\begin{tabular}{|c|c|c|c|c|c|}
\hline \multirow[t]{2}{*}{ Año } & \multicolumn{2}{|c|}{$\begin{array}{c}\text { Inadecuado material de } \\
\text { pisos }\end{array}$} & \multicolumn{2}{|c|}{$\begin{array}{c}\text { Inadecuado material de } \\
\text { paredes exteriores }\end{array}$} & \multirow[t]{2}{*}{ TOTAL } \\
\hline & No privación & Privación & $\begin{array}{l}\text { No } \\
\text { privación }\end{array}$ & Privación & \\
\hline 2013 & $\begin{array}{r}11.417 .803 \\
(93,7 \%) \\
\end{array}$ & $\begin{array}{r}773.104 \\
(6,3 \%) \\
\end{array}$ & $\begin{array}{r}11.905 .806 \\
(97,7 \%) \\
\end{array}$ & $\begin{array}{r}285.101 \\
(2,3 \%) \\
\end{array}$ & $\begin{array}{r}12.190 .908 \\
(100 \%) \\
\end{array}$ \\
\hline 2014 & $\begin{array}{r}11.728 .057 \\
(94,2 \%) \\
\end{array}$ & $\begin{array}{r}716.418 \\
(5,8 \%) \\
\end{array}$ & $\begin{array}{r}12.202 .845 \\
(98,1 \%) \\
\end{array}$ & $\begin{array}{r}241.629 \\
(1,9 \%) \\
\end{array}$ & $\begin{array}{r}12.444 .475 \\
(100 \%) \\
\end{array}$ \\
\hline 2015 & $\begin{array}{r}11.808 .403 \\
(94,4 \%) \\
\end{array}$ & $\begin{array}{r}696.416 \\
(5,6 \%) \\
\end{array}$ & $\begin{array}{r}12.218 .016 \\
(97,7 \%) \\
\end{array}$ & $\begin{array}{r}286.804 \\
(2,3 \%)\end{array}$ & $\begin{array}{r}12.504 .820 \\
(100 \%) \\
\end{array}$ \\
\hline 2016 & $\begin{array}{r}12.469 .095 \\
(93,4 \%) \\
\end{array}$ & $\begin{array}{r}880.410 \\
(6,6 \%) \\
\end{array}$ & $\begin{array}{r}12.811 .250 \\
(96 \%) \\
\end{array}$ & $\begin{array}{r}538.255 \\
(4 \%) \\
\end{array}$ & $\begin{array}{r}13.349 .505 \\
(100 \%)\end{array}$ \\
\hline
\end{tabular}

Fuente: Elaboración propia con microdatos del Dane

Por último, y relacionando los hogares que reportan privación por hacinamiento crítico, fue el año 2013 con 13,6\%. Ver Tabla No 7.

Tabla No 7: Servicios públicos y condiciones de vivienda

\begin{tabular}{|r|r|r|r|}
\hline Año & \multicolumn{2}{|c|}{ Hacinamiento crítico } & \multirow{2}{*}{ TOTAL } \\
\hline & No privación & Privación & \\
\hline \multirow{2}{*}{2013} & 10.530 .409 & 1.660 .499 & 12.190 .908 \\
& $(86,4 \%)$ & $(13,6 \%)$ & $(100 \%)$ \\
\hline 2014 & 10.995 .570 & 1.448 .904 & 12.444 .475 \\
& $(88,4 \%)$ & $(11,6 \%)$ & $(100 \%)$ \\
\hline 2015 & 11.231 .836 & 1.272 .984 & 12.504 .820 \\
& $(89,8 \%)$ & $(10,2 \%)$ & $(100 \%)$ \\
\hline 2016 & 11.952 .212 & 1.397 .293 & 13.349 .505 \\
& $(89,5 \%)$ & $(10,5 \%)$ & $(100 \%)$ \\
\hline
\end{tabular}

Fuente: Elaboración propia con microdatos del Dane

Modelo Lineal Generalizado. Regresión Beta

Variables tales como las tasas, las proporciones y los datos fraccionarios, son con frecuencia entre cero y uno. Estos exigen una variedad de métodos dentro de los que se encuentran la regresión beta y regresión logística fraccional. Estos tipos de regresiones son ampliamente utilizadas gracias a su flexibilidad para modelar variables entre 0 y 1 y porque sus predicciones están confinadas al mismo rango.

En este sentido, Ferrari y Cribari-Nieto (2004) han propuesto un modelo de regresión para variables que tienen una distribución beta, muy flexible para modelar proporciones, ya que su densidad puede tener formas bastante diferentes dependiendo de los valores de los dos parámetros que indexan la distribución. La distribución de densidad beta tiene dos parámetros p y q y está dada por: 


$$
\pi(y, p, q)=\frac{\Gamma(p+q)}{\Gamma(p) \Gamma(q)} y^{p-1}(1-y)^{q-1} \quad \operatorname{con} 0<y<1
$$

donde $p>0, q>0 y \Gamma(\cdot)$ es la función gamma.

Sin embargo, para el análisis de regresión, generalmente es más útil modelar la media de la respuesta. También, es típico definir el modelo para que contenga un parámetro de precisión (o dispersión). Para obtener una estructura de regresión para la media de la respuesta junto con un parámetro de precisión, trabajaremos con una parametrización tal que:

$$
\mu=\frac{p}{p+q}, \phi=p+q \text {, e decir que } p=\mu \phi \text { y } q=(1-\mu) \phi
$$

de modo que $\mu$ es la media de la variable de respuesta y $\phi$ puede interpretarse como un parámetro de precisión en el sentido de que, para $\mu$ fijo, cuanto mayor sea el valor de $\phi$, menor la varianza de $y$. La densidad de $y$ se puede escribir en la nueva parametrización como:

$$
\pi(y ; \mu, \phi)=\frac{\Gamma(\phi)}{\Gamma(\mu \phi) \Gamma((1-\mu) \phi)} y^{\mu \phi-1}(1-y)^{(1-\mu) \phi-1}, \quad 0<y<1
$$

donde $0<\mu<1, \phi>0$

La estimación de los dos parámetros $\mu, \phi$ desconocidos se realizan por máxima verosimilitud.

Las estimaciones de los modelos para cada uno de los años fueron realizadas en el software estadístico R.

Como producto, en los cuatro años del periodo de estudio, resultan ser significativas 12 variables de las incluidas. Las que no, están relacionadas con: el número de cuartos en la vivienda, la región, el material de las paredes exteriores y el saber leer y estar estudiando por parte del jefe de hogar.

De las variables que resultaron significativas, para la variable "personas" su coeficiente se reporta positivo. Se evidencia que, al aumentar el número de personas del hogar, también aumenta el IPM y la variable Edad de jefe de hogar muestra un coeficiente negativo, lo que indica que un aumento en la edad del jefe del hogar genera una reducción del índice para el hogar. Además, para las variables restantes (10) que están relacionadas con las dimensiones también se muestra que al estar privado por alguna de las condiciones mencionadas anteriormente incrementa el Índice de Pobreza Multidimensional.

Efectos Marginales

Los efectos marginales constituyen la proporción e influencia de un cambio en las variables exógenas en el índice de pobreza multidimensional. Para el periodo 2013-2016, 
en promedio y céteris páribus, para las privaciones directamente relacionadas con la vivienda se tiene que:

El tener privación por agua incrementó el IPM de que un hogar en 0,03 unidades, por alcantarillado en 0,04 unidades, por inadecuado material de pisos en 0,03 unidades, por inadecuado material de paredes exteriores en 0,04 unidades, por hacinamiento en 0,03 unidades.

Con relación a las características de hogar, la que mayor tiene efecto es la privación por logro educativo, con un incremento de 0,10 . Ver Tabla No 8.

Tabla No 8: Efectos Marginales

\begin{tabular}{|c|c|c|c|c|}
\hline Variable & $\begin{array}{c}\text { Dy/Dx } \\
2013\end{array}$ & Dy/Dx 2014 & $\begin{array}{c}\text { Dy/Dx } \\
2015\end{array}$ & Dy/Dx 2016 \\
\hline PERSONAS & 0,0110 & 0,0105 & 0,0109 & 0,0105 \\
\hline $\begin{array}{c}\text { LOGRO_ED } \\
\text { (Privación) }\end{array}$ & 0,1184 & 0,1187 & 0,1167 & 0,1151 \\
\hline $\begin{array}{c}\text { INASISTE } \\
\text { (Privación) }\end{array}$ & 0,0684 & 0,0740 & 0,0752 & 0,0665 \\
\hline $\begin{array}{c}\text { DESEMPLE } \\
\text { (Privación) }\end{array}$ & 0,1080 & 0,1106 & 0,1085 & 0,1058 \\
\hline $\begin{array}{c}\text { ASEGURAM } \\
\text { (Privación) }\end{array}$ & 0,1154 & 0,1109 & 0,1104 & 0,1105 \\
\hline $\begin{array}{c}\text { ACUEDUCT } \\
\text { (Privación) }\end{array}$ & 0,0390 & 0,0393 & 0,0365 & 0,0386 \\
\hline $\begin{array}{c}\text { ALCANTAR } \\
\text { (Privación) }\end{array}$ & 0,0408 & 0,0380 & 0,0398 & 0,0355 \\
\hline PISOS (Privación) & 0,0378 & 0,0379 & 0,0353 & 0,0397 \\
\hline $\begin{array}{c}\text { PAREDES } \\
\text { (Privación) }\end{array}$ & 0,0440 & 0,0402 & 0,0401 & 0,0381 \\
\hline $\begin{array}{c}\text { HACINAMI } \\
\text { (Privación) }\end{array}$ & 0,0315 & 0,0369 & 0,0342 & 0,0351 \\
\hline P6040 & $-0,0001$ & $-0,0002$ & $-0,0002$ & $-0,0001$ \\
\hline $\begin{array}{c}\text { P6920 } \\
\text { No }\end{array}$ & 0,0608 & 0,0619 & 0,0617 & 0,0612 \\
\hline Pensionado & 0,0173 & 0,0190 & 0,0160 & 0,0209 \\
\hline
\end{tabular}

Fuente: Elaboración propia con microdatos del Dane

Para evaluar modelos estadísticos, dos criterios usados y aceptados de modo más amplio para la selección de modelos son el Criterio de Información de Akaike (AIC) y el criterio Schwarz Bayesiano (BIC).

El primero, enfatiza en la bondad de ajuste del modelo, en cuanto a que un valor más pequeño establece un mejor ajuste del modelo. Ante ello, Caballero (2011) afirma que algunas de las ventajas del AIC, que lo hacen tan utilizado en la práctica, son su simplicidad (no requiere acudir a ninguna tabla para observar el valor correspondiente) 
y facilidad para ser implementado, y el hecho de que no existe el problema de especificar subjetivamente un nivel de significación arbitrario para contrastar dos modelos.

En segundo (BIC), a diferencia del AIC, considera el número n de observaciones en el término de penalización, y es además menos favorable a la inclusión de factores. El procedimiento de selección de un modelo factorial en base al BIC será selecciona el modelo con un número de factores, $\mathrm{m}$, tal que se minimice el valor del BIC.

En los dos criterios y para cada uno de los años del periodo los valores son negativos, lo cual muestra un valor mínimo dando confianza al que el modelo trabajado es adecuado. La situación inversa ocurre con el clásico Pseudo $R^{2}$, que refleja una buena relación entre las variables incluidas en el modelo y el IPM para cada año del estudio. Ver Tabla No 9 .

Tabla No 9: $A I C, B I C$, Pseudo $R^{2}$

\begin{tabular}{|c|c|c|c|}
\hline Años/Criterios & AIC & BIC & Pseudo $R^{2}$ \\
\hline 2013 & -55.519 & -54.924 & 0,7965 \\
\hline 2014 & -59.953 & -59.687 & 0,7931 \\
\hline 2015 & -67.731 & -67.453 & 0,7892 \\
\hline 2016 & -68.801 & -68.525 & 0,7894 \\
\hline
\end{tabular}

Fuente: Elaboración propia con microdatos del Dane

\section{Sección 4: Recomendaciones para política pública}

La vivienda es una posesión fundamental que hace parte de la riqueza inmobiliaria de los hogares en Colombia y su tenencia suele representar bienestar. Las soluciones que se han propuesto desde la política pública para los problemas de vivienda en Colombia han sido diversas. La intervención del estado mediante subsidios fomenta la adquisición de $\mathrm{v}$ vivienda en los sectores público y privados; sin embargo, muchas de estas soluciones han sido momentáneas y en aras de cumplir un plan de desarrollo de cuatro años o un gobierno de ocho, pero no necesariamente se han convertido en políticas de Estado que logren una eficaz trascendencia a lo largo del tiempo.

A la luz de los resultados empíricos de esta investigación, la tenencia de vivienda no es la característica de los hogares que más impacta el índice de pobreza multidimensional. Por esta razón, es importante fijar el interés también en este aspecto, pues el hecho de contar con un techo y un abrigo adecuado genera seguridad que se ve reflejada también en la confianza de los individuos de una sociedad para asumir nuevos retos de desarrollo. Respecto a ¿cuáles han sido los efectos de la tenencia de vivienda y algunas características de los hogares en el Índice de Pobreza Multidimensional para Colombia en el periodo 2013-2016? Al establecer como variable dependiente el Índice de Pobreza multidimensional y como variables independientes las 17 planteadas en la tabla No 1 de esta investigación, se encuentra que no todas resultan significativas, tal como se evidencia en los anexos A, B, C, y D, para una modelación bajo una regresión beta. 
Las variables relacionadas con la tenencia de vivienda en cuanto a la dimensión servicios públicos, acueducto y alcantarillado y condiciones de la vivienda, material de pisos, paredes y hacinamiento todas tienen un efecto positivo dentro del IPM; al estar los hogares dentro de las privaciones allí expuestas, su Índice de Pobreza Multidimensional se hace mayor.

Respecto a las variables acerca de las características de hogar, las que tienen un mayor efecto sobre el índice son las privaciones por logro educativo, por aseguramiento en salud y por desempleo, lo cual indica que, en procura de una reducción del índice de pobreza multidimensional, se deben poner en marcha apuestas y programas para cada uno de estos sectores, de forma equilibrada. De modo que para el desarrollo humando de los individuos estas oportunidades de mejora actúen de forma complementaria.

Ahora bien, estas apuestas políticas deben estar mediadas por el gobierno con la conciencia de que al fomentar que los ciudadanos tengan vivienda propia se estaría incentivando la reducción de índices de vulnerabilidad como el Índice de Pobreza Multidimensional que aborda esta investigación. Esto es, que la vivienda no puede convertirse en un pasivo a largo plazo, pues de igual manera esta debe contar con las condiciones mínimas de habitabilidad, unas que podrían ser solucionadas por los individuos, como parte de la estructura y otras que sin duda las debería garantizar en su totalidad el gobierno, como la accesibilidad a los servicios públicos.

En esta dirección y con relación al tema de los subsidios, es importante mencionar que estos han venido beneficiando el acceso a la vivienda a muchos hogares; sin embargo, a otros los han limitado, en la medida que solo aplican para un determinado rango de ingresos familiares y precio del inmueble. Luego, si el interés y la realidad de un hogar se sale de estas bandas, no podrá tener acceso a este tipo de beneficios.

Algunos de estos programas lastimosamente se toman como solución de momento, sin garantía de continuidad a lo largo del tiempo, ocasionando así que muchos ciudadanos opten por no acceder a ellos. En el caso de la tenencia de vivienda, los hogares deben contar con un monto de dinero para ello, pues así sean beneficiarios de subsidios, no todo es cubierto por todos los programas y muchas veces la falta de pago de créditos hace que este lugar se pierda o la falta de mantenimiento o deterioro de la vivienda hace que el lugar adquirido no sea el adecuado para habitarlo. Sin embargo, actualmente existen aún partes del territorio colombiano donde la falta de presencia del estado es bastante evidente, obras inconclusas que generan una falta de agua y alcantarillado, por ejemplo, dejando de lado el bienestar de los individuos.

No obstante, es muy importante seguir impulsando estos subsidios y cada vez ampliarlos más, de modo que en el territorio nacional más hogares puedan acceder a ellos en pro de tener una vivienda propia o mejorar la que ya se tiene.

También, es vital recomendar que gran parte del éxito de las políticas habitacionales está determinado por recursos por parte del gobierno para el cumplimiento y continuidad de las mismas, teniendo en cuenta que estas deben ser encauzadas a formar y desarrollar las capacidades básicas de las personas, ya que atienden a las necesidades básicas de los más vulnerables, que, por diversas circunstancias, tienen dificultades para abastecerse de manera autónoma y procurar su desarrollo humano individual. A continuación, se muestra la distribución de este gasto social en el tiempo de interés de ésta investigación. 


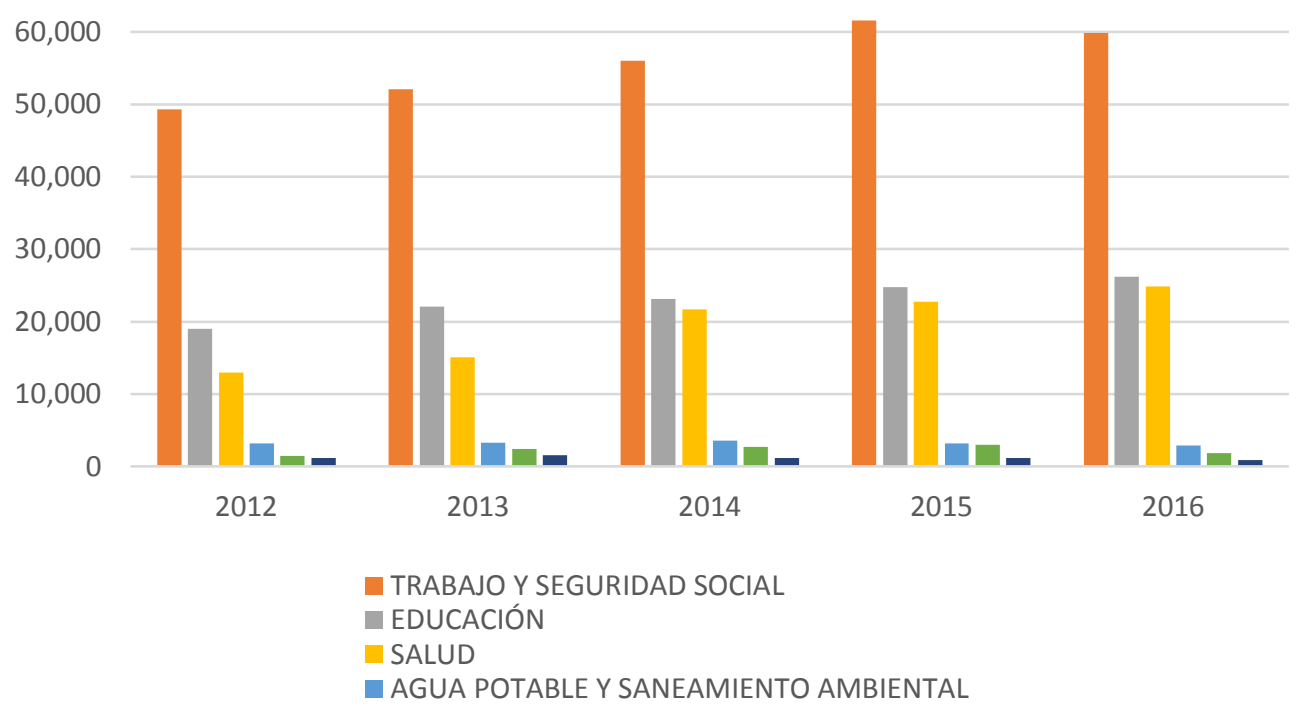

Figura No 2: Distribución del gasto Social

Fuente: Elaboración propia con datos del Ministerio de Hacienda

Aquí se observa que la mayor cantidad de recursos han sido destinados desde el gasto social para las funciones Trabajo y Seguridad Social y lo que respecta a la Vivienda Social se encuentra en el penúltimo lugar. El año 2015 es el año con la mayor cantidad de miles de millones 3.004 de gasto. Ahora bien, por cifras del observatorio social de la CEPAL (Comisión Económica para América Latina y el Caribe), el porcentaje del PIB, para la función vivienda se encuentra en promedio en un $0.5 \%$, mientas que la función protección social supera en promedio los 3.5 puntos porcentuales.

Esta comisión también refrenda la preocupación de que en el país se destinan 400 dólares por habitante, mientras que en el continente el promedio es de 1.841 dólares por persona.

En esta dirección, se deberían implementar mecanismos de equidad para que todas las funciones tuvieran unos recursos más equilibrados. Si bien es cierto las dimensiones de la pobreza son múltiples, las necesidades de los individuos también lo son. En este sentido «la importancia que para el desarrollo tiene el uso amplio de los mercados, así como el papel singular del apoyo social, de la regulación pública o la política cuando pueden enriquecer, en lugar de empobrecer, vidas humanas» (Sen, 2000, p. 19).

Ahora bien, se puede establecer, en palabras de Justo y Velásquez (2000), que el stock de vivienda de cada país, con sus características de formas de promoción y tenencia, tamaño, antigüedad, así como el déficit cuantitativo y cualitativo, son resultado de procesos y políticas determinadas en contextos históricos y variables comunes: la demanda, función del crecimiento vegetativo de la población, las migraciones internas y externas, la situación económica, el gasto público, el nivel de ingresos de la población, la acumulación de la riqueza y su distribución, la pobreza y los bajos ingresos, la 
financiación directa e indirecta por parte del Estado y los privados, las políticas de arrendamiento o de acceso a la propiedad.

Finalmente, se considera que el dar a los hogares la posibilidad de ser propietario de una vivienda, traería consigo beneficios que a largo plazo se verían reflejados, en primer lugar, en incentivar una movilidad social que, junto con el acceso a la educación, según lo mostrado en esta investigación, lograría un mejor desempeño en términos económicos en sociedades en vía de desarrollo. En segundo lugar, aportaría en gran medida al bienestar tanto de forma individual (personas-hogares), como colectivo (hogares-país), propiciando así tranquilidad, felicidad y realización en los ciudadanos, alejándolos de estados de vulnerabilidad y/o pobreza.

El bienestar de los individuos u hogares se relaciona de forma consecuente a lo planteado en el marco teórico. Por un lado, hacia lo que refiere Sen en su teoría de Desarrollo Humano, donde involucra la capacidad, oportunidad y libertad, que se le brinda a un individuo para que haga de su vida una vida placentera y digna de vivir. Aquí las oportunidades de educación, salud y vivienda fundamentarían la consecución de personas con mejor calidad de vida, en menor riesgo de ser vulnerables o caer en la pobreza multidimensional. Por otro lado, y en relación con las Necesidades Básicas Insatisfechas, es claro que la falta de atención por parte del los gobiernos en minimizar estas necesidades se ve reflejada en el efecto tan marcado de algunas de las variables hacia el IMP; lo que Max-Neef reconoce como las "pobrezas" relacionadas a la subsistencia, protección, afecto, entendimiento, participación, identidad, ocio, creación y libertad, de las cuales esta investigación hace referencia a la subsistencia, protección y afecto.

Recibido: 2019-02-24

Aceptado: 2019-06-17

\section{Referencias}

Álvarez, M. y Martínez, H. (2001). El desafío de la pobreza. Bogotá D.C., Colombia: Siglo del hombre.

Angulo R, Díaz y, Pardo R. (2011) Índice de Pobreza Multidimensional para Colombia (IPM-Colombia) 1997-2010. Colombia: Archivos de Economía.

Alkire, S. (2002). Dimensions of human development. World Development, 30(2), 181205. doi: $10.1016 / \mathrm{S} 0305-750 \mathrm{X}(01) 00109-7$

Alkire, S., \& Foster, J. (2011). Understandings and misunderstandings of multidimensional poverty measurement. Journal of Economic Inequality, 9(2), 289-314. doi: 10.1007/s10888-011-9181-4 
Alkire, S., \& Foster, J. (2011). Counting and multidimensional poverty measurement. Journal of Public Economics, 95(7-8), 476-487. doi:

10.1016/j.jpubeco.2010.11.006

Bedoya, C (2010). Amartya Sen y el desarrollo humano. Revista Nacional de Investigación Memorias, 8(13), 277-288.

Caballero Díaz, F. F. (2011). Selección de Modelos Mediante Criterios de Información en Análisis Factorial. Aspectos teóricos y Computacionales. Universidad de Granada. Recuperado de http://dialnet.unirioja.es/servlet/tesis?codigo=22590

Camargo, A. (2011). Vivienda y pobreza: una relación compleja. Cuadernos De Vivienda y Urbanismo. 4(8), 224-246.

Cejudo Córdoba, R. (2007). Capacidades y libertad: una aproximación a la teoría de Amartya Sen, Revista Internacional de Sociología, 65(47), 9-22.

Congreso de la República de Colombia (8 de febrero de 1994). Ley 115. Ley General de educación. Recuperado de https://www.mineducacion.gov.co/1621/articles85906_archivo_pdf.pdf

Decreto 3011 de diciembre 19 de 1997.

Comunicado de prensa. Pobreza en Colombia. (2012) Departamento Nacional de Estadísticas (DANE). Colombia.

Encuesta nacional de hogares. (s.f.) Departamento Nacional de Estadísticas (DANE).

Ferrari SLP, Cribari-Neto F (2004). Beta Regression for Modelling Rates and Proportions. Journal of Applied Statistics, 31(7), 799-815.

Fondo Nacional de Vivienda. FONVIVIENDA. Resolución 053520 de Junio de 2012.

Recuperado de: http://www.minvivienda.gov.co/ResolucionesVivienda/0535\%20\%202012.pdf

Max-Neef, M. (1993). Desarrollo a escala humana. Icaria, Montevideo.

Maya, E. y Bournazou, E. (2012). La vivienda en México. Temas con Temporáneos, pp 143: Facultad de Arquitectura y Dirección General de Personal Académico unam. México. isbn: 978-607-02-3179-7.

PNUD. Desarrollo Humano: Informe 1992. Bogotá - Colombia. Tercer Mundo

PNUD. Desarrollo Humano: Informe 1990. Bogotá - Colombia. Tercer Mundo 
Ruprah, I. (2010). Do Social Housing Programs Increase Poverty? An Empirical Analysis of Shelter Induced Poverty in Latin America. OVE Working Papers 0510, Inter-American Development Bank, Office of Evaluation and Oversight (OVE).

Salinas-Rodríguez, A. (2006). Modelos de regresión para variables expresadas como una proporción continua. Revista Salud Pública de México, 48(5), 395-404.

Recuperado de: http://www.scielo.org.mx/scielo.php?pid=S0036$36342006000500006 \&$ script $=$ sci_abstract.

Sen, A. (2000). Desarrollo y Libertad (E. Rabasco y L. Toharia, Trad.). Barcelona.: Planeta S.A. (Versión original publicada en 1999)

Villarroel, Paul \& Hernani-Limarino, Werner (2013) La evolución de la pobreza en Bolivia: un enfoque multidimensional. Revista Latinoamericana de Desarrollo Económico, Instituto de Investigaciones Socio-Económicas (IISEC), Universidad Católica Boliviana, 20(noviembre), 7-75. 